\title{
A new technique of acoustic mode filtering in shallow sea
}

\author{
Harish M. Chouhan and G. V. Anand \\ Department of Electrical Communication Engineering. Indian Institute of Science, \\ Bangalore 560012, India
}

(Received 26 May 1989; revised 17 August 1990; accepted 23 October 1990)

\begin{abstract}
A new technique of normal mode filtering in an isovelocity channel using an equispaced linear array is presented. Filtering is achieved by steering nulls in the directions of arrival of the quasiplane wave pairs associated with the normal modes that are to be rejected. A theoretical analysis of the problem is presented for both horizontal and nonhorizontal arrays. The minimum number of array elements and their weighting coefficients are determined for each case. The null-steering technique offers several advantages over other techniques of mode filtering. The weighting coefficients are independent of the array depth. A horizontal or vertical array of $2 M-1$ elements with real weighting coefficients or a horizontal array of $M$ elements with complex weighting coefficients is sufficient to select one mode from a set of $M$ modes. There is no constraint on interelement spacing. The filter performance is equally good under all types of bottom conditions. If a horizontal array is deployed, this method of mode filtering can be used in a horizontally stratified channel also. The null-steering method gives perfect filtering if the orientation of the array and the modal wave numbers are known exactly. An analysis of the sensitivity of the mode filter to errors in the assumed values of these parameters is also presented.
\end{abstract}

PACS numbers: 43.30.Wi, 43.30.Bp

\section{INTRODUCTION}

The acoustic field in a water channel can be represented by a sum of normal modes of the channel. The normal mode parameters are dependent on the acoustic properties of the channel and the source parameters. Recently there has been considerable interest in the efficient filtering of the normal modes. ${ }^{1-10}$ This is due to the fact that mode filtering techniques can be used to determine the acoustic properties of the channel..$^{3,4}$ On the other hand, if the acoustic properties of the channel are known, these techniques can be used for source localization. ${ }^{4.9}$ Mode filtering has also found application in ocean acoustic tomography. ${ }^{10}$

In a shallow water channel of constant depth $h$, constant density $\rho$, and sound speed $c(z)$ overlying a bottom of density $\rho_{1}$, and sound speed $c_{1}(z)$, the acoustic pressure in the far field of a point source of angular frequency $\omega$ can be written as

$$
p(r, z, t)=\sum_{m=1}^{M} p_{m}(r, z) \exp \left[-j\left(\omega t-\frac{\pi}{4}\right)\right],
$$

where

$$
p_{m}(r, z)=\left(2 \pi / k_{m} r\right)^{1 / 2} \psi_{m}\left(z_{1}\right) \psi_{m}(z) \exp \left(j k_{m} r\right) .
$$

In Eq. (2) $z_{s}$ is the source depth, $z$ is the receiver depth, $r$ is the range, and $\psi_{m}(z)$ are a set of orthonormal eigenfunctions satisfying the condition

$$
\int_{0}^{h} \rho^{-1} \psi_{m}(z) \psi_{n}(z) d z+\int_{h}^{\infty} \rho_{1}^{-1} \psi_{m}(z) \psi_{n}(z) d z=\delta_{m n} .
$$

\section{Defining}

$$
k(z)=\omega / c(z),
$$

the horizontal component $k_{m}$ and the vertical component $\gamma_{m}$ of the $m$ th mode wave number are related as

$$
k^{2}(z)=k_{m}^{2}+\gamma_{m}^{2}(z)
$$

Time resolution ${ }^{11}$ as well as spatial processing techniques $^{12,13}$ have been used for mode filtering. Spatial processing techniques use a steered horizontal array ${ }^{12}$ or a vertical array with a suitable mode filtering processor. ${ }^{1-3,14,15}$ The mode-matching technique of mode filtering utilizes the orthogonality property of the normal mode eigenfunctions as stated in Eq. (3). In this technique, a discrete vertical array of $Q$ hydrophones is used. With the shading coefficients set proportional to $\psi_{m}\left(z_{q}\right)$, where $z_{q}$ is the depth of the $q$ th hydrophone, the output of the array due to the $n$th mode is given by

$$
I_{m n}(r)=B \sum_{q=1}^{Q} \psi_{m}\left(z_{q}\right) p_{n}\left(r, z_{q}\right)
$$

Substituting for $p_{n}\left(r, z_{q}\right)$ from Eq. (2), we get

$$
\begin{aligned}
I_{m u}(r)= & B a_{n} \psi_{n}\left(z_{s}\right)\left(\frac{2 \pi}{k_{u} r}\right)^{1 / 2} \exp \left(j k_{n} r\right) \\
& \times \sum_{q=1}^{Q} \psi_{m}\left(z_{q}\right) \psi_{n}\left(z_{q}\right) .
\end{aligned}
$$

Comparison of Eq. (3) and Eq. (6) indicates that $I_{m n} \simeq 0$ for $n \neq m$. But this technique has several disadvantages. A large number of hydrophones and their judicious positioning is required to achieve satisfactory filtering. Furthermore the array has to span the entire depth where the eigenfunctions have an appreciable amplitude. Tindle $e t a l .^{2}$ have proposed a least-squares method that does not require a large number of hydrophones. But both these techniques are sensitive to vertical displacement of the array. A new technique that does not suffer from these drawbacks is presented in the following sections. 


\section{FORMULATION OF THE PROBLEM}

Consider a linear hydrophone array of $2 Q+1$ identical omnidirectional elements in the far field of a point source in an isovelocity shallow water channel. The geometry of the problem is shown in Fig. 1.

The array is located in the $x z$ plane making an angle $\alpha$ with the $z$ axis and the coordinates of the elements are $\left(x_{q}, 0, z_{q}\right), q=-Q,-Q+1, \ldots, 0, \ldots, Q-1, Q$, with $x_{0}=0$. The source is located at $\left(x_{s}, y_{s}, z_{s}\right)$, and

$$
\beta=\tan ^{-1}\left(y_{s} / x_{s}\right)
$$

denotes the source bearing angle. Denoting the horizontal range of the element $q$ from the source by $r_{q}$, we have (see Fig. 1)

$$
r_{q}=\left(r^{2}+x_{q}^{2}-2 r x_{q} \cos \beta\right)^{1 / 2},
$$

where $r=r_{0}$ is the horizontal range of the central element. If $r \gg\left(x_{Q}-x_{-Q}\right)$, the projected array length in the $x y$ plane, we get the approximation

$$
r_{q}=r-x_{q} \cos \beta
$$

If the array has uniform interelement spacing $d$, we can write

$$
x_{q}=q d \sin \alpha, \quad z_{q}=q d \cos \alpha+z_{0} .
$$

Assuming the array to be in an isovelocity water layer, the sound pressure $p_{q}$ at the $q$ th element can be expressed as

$$
\begin{aligned}
p_{q}= & \sum_{m=1}^{M} a_{m}^{2}\left(\frac{2 \pi}{k_{m} r_{q}}\right)^{1 / 2} \sin \left(\gamma_{m} z_{s}\right) \sin \left(\gamma_{m} Z_{q}\right) \\
& \times \exp \left[j\left(k_{m} r_{q}+\pi / 4\right)\right],
\end{aligned}
$$

where $a_{m}$ is the normalization constant for the $m$ th mode, and the harmonic time dependence is suppressed. The array output, obtained by weighting the hydrophone outputs and summing them, is given by

$$
R=\sum_{q=-Q}^{Q} w_{q} p_{q},
$$

where the weighting coefficients $w_{q}$ may be complex. Using the approximation given by Eq. (9) in the phase term of Eq. (11) and the approximation $r_{q} \simeq r$ in the amplitude term, we can write Eq. (12) as

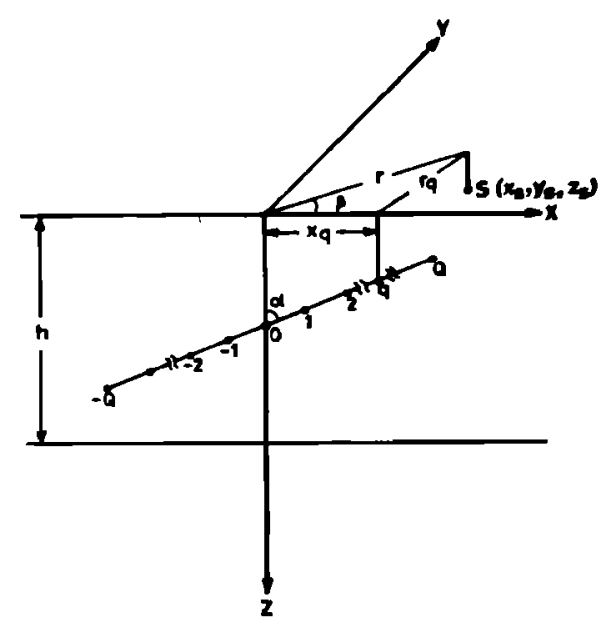

FIG. 1. Geometry of the receiver array and the source.

$$
\begin{aligned}
R= & \sum_{q=-Q}^{Q} \sum_{m=1}^{M} w_{q} a_{m}^{2}\left(\frac{2 \pi}{k_{m} r}\right)^{1 / 2} \sin \left(\gamma_{m} z_{s}\right) \sin \left(\gamma_{m} z_{q}\right) \\
& \quad \times \exp \left\{j\left[k_{m}\left(r-x_{q} \cos \beta\right)+\pi / 4\right]\right\} .
\end{aligned}
$$

Interchanging the order of summation in the above expression, we have

$$
R=\sum_{m=1}^{M} g_{m} A_{m},
$$

where

$A_{m}=a_{m}\left(2 \pi / k_{m} r\right)^{1 / 2} \sin \left(\gamma_{m} z_{s}\right) \exp \left[j\left(k_{m} r+\pi / 4\right)\right]$,

is the complex amplitude of the $m$ th mode and

$g_{m}=\sum_{q=-Q}^{Q} a_{m} w_{q} \sin \left(\gamma_{m} z_{q}\right) \exp \left(-j k_{m} x_{q} \cos \beta\right)$,

is the array gain factor for the $m$ th mode.

The problem of mode filtering, i.e., selecting a specified normal mode $n$ while rejecting all the others, can be stated as follows: find the sequence of weighting coefficients $W_{n}$ $=\left\{w_{-Q}, w_{-Q+1}, \ldots, w_{Q}\right\}$ such that

$$
g_{m}=0, \quad m \neq n \text {. }
$$

In an isovelocity water channel, each normal mode is formed by the interference of two quasiplane waves propagating symmetrically with respect to the horizontal plane. Hence, a mode can be rejected by steering nulls of the array response in the directions of arrival of the associated pair of plane waves. Let the sequence of weighting coefficients required to steer nulls in the directions of arrival of the plane waves associated with the $m$ th mode be denoted by $U_{m}$. In order to filter the $n$th mode and reject all the others, it is necessary to design an array whose response has nulls in the directions of arrival of all of the unwanted modal plane wave pairs. If the channel contains $M$ modes, the sequence of weighting coefficients of the $n$th mode filter is therefore given by the convolution of $M-1$ elementary sequences $U_{m}, m \neq n$,

$$
W_{n}=U_{1} * U_{2} * \cdots * U_{n-1} * U_{n+1} * \cdots * U_{m},
$$

where $*$ denotes convolution. Expressions for the elementary sequence $U_{m}$ under various conditions are derived in the following sections.

\section{MODE FILTERING WITH A HORIZONTAL ARRAY}

For an equispaced horizontal array having an interelement spacing $d$ and located at depth $z_{0}$, we have

$$
z_{q}=z_{0}, \quad x_{q}=q d .
$$

The array gain factor for mode $m$, obtained from Eq. (16), can now be written as

$g_{m}=a_{m} \sin \left(\gamma_{m} z_{0}\right) \sum_{q=-Q}^{Q} w_{q} \exp \left(-j k_{m} q d \cos \beta\right)$.

The gain factor is dependent on the array depth $z_{0}$ and becomes zero if $z_{0}$ is the depth at which the $m$ th mode function has a node. We assume this is not the case. Let the directions of arrival of the $m$ th modal plane waves with respect to the array axis be denoted by $\theta_{m 1}$ and $\theta_{m 2}$. For a horizontal array $\theta_{m 1}$ and $\theta_{m 2}$ are equal, and are given by 


$$
\left.\theta_{m 1}=\theta_{m 2}=\theta_{m}=\cos ^{-1}\left[\left(k_{m} / k\right) \cos \beta\right)\right] .
$$

Hence mode $m$ can be rejected by steering a single null of the array response in the direction $\theta_{m}$. The arrival directions and hence the interarrival angular separations are functions of $\beta$. For a source in the endfire direction, i.e., $\beta=0$, we have maximum separation amongst the different arrivals. With increasing $\beta$, the arrivals get more and more clustered. When the source is in the broadside direction, i.e., when $\beta=\pi / 2$, all the arrival angles are equal to $\pi / 2$ and mode filtering is not possible.

In order to determine the weights required to reject the $m$ th mode, we consider the array response to mode $m$. From Eq. (20) we see that $g_{m}=0$ if the weights $w_{q}$ are chosen as follows:

$$
\begin{array}{ll}
w_{p+1} / w_{p}=-\exp \left(j k_{m} d \cos \beta\right), & \text { for some } p, \\
w_{q}=0, & \text { for } q \neq p \text { or } p+1 .
\end{array}
$$

Hence, mode $m$ can be rejected by employing a two-element array whose weights are given by the sequence

$$
U_{m}^{H}=\left\{1,-\exp \left(j k_{m} d \cos \beta\right)\right\},
$$

where the superscript $H$ denotes that the array is horizontal. The weight sequence $W_{n}^{H}$ of a horizontal $n$th mode filter is obtained by substituting $U_{m}=U_{m}^{H}(m=1, \ldots, n-1, n$ $+1, \ldots, M)$ in Eq. (18). Since $U_{m}^{H}$ contains two elements, the sequence $W_{n}^{H}$ contains $M$ elements.

The response of a weighted equispaced linear array to a plane wave incident at an angle $\theta$ with respect to the endfire direction is given by

$$
\boldsymbol{B}(\theta)=\sum_{q} w_{q} \exp (-j q k d \cos \theta) .
$$

Hence the free-field directivity function of the array whose weighting coefficients are given by the sequence $U_{m}^{H}$ of Eq. (23) is given by

$$
B_{m}(\theta)=1-\exp \left[j d\left(k_{m} \cos \beta-k \cos \theta\right)\right] .
$$

As expected, the free-field directivity function has a null at $\theta_{m}=\cos ^{-1}\left[\left(k_{m} / k\right) \cos \beta\right]$, which is the direction of arrival of the modal waves of mode $m$. The free-field directivity function of the $n$th mode filter with weight sequence $W_{n}^{H}$ is given by

$$
D_{n}(\theta)=\prod_{\substack{m \\ m \neq n}}^{M} B_{m}(\theta) .
$$

It has been shown that mode $m$ can be rejected by employing a two-element array with a complex weight sequence $U_{m}^{H}$. The same function can also be accomplished using a three-element array with a real weight sequence $U_{m}^{H R}$ given by

$$
U_{m}^{H R}=U_{m}^{H} * U_{m}^{H \bullet},
$$

where $U_{m}^{H}$ • denotes a sequence whose elements are the complex conjugates of the elements of $U_{m}^{H}$. Substituting Eq. (23) in Eq. (27), we get

$$
U_{m}^{H R}=\left\{1,-2 \cos \left(k_{m} d \cos \beta\right), 1\right\} .
$$

The array defined by Eq. (28) is real and symmetric and steers zeros simultaneously in the directions $\theta_{m}$ and $\pi-\theta_{m}$.
Hence filtering of mode $n$ can be achieved by a $(2 M-1)$ element real symmetric array with a weight sequence $W_{n}^{\prime \prime R}$ obtained by substituting $U_{m}=U_{m}^{H R}(m=1, \ldots, n-1, n$ $+1, \ldots, M)$ in Eq. (18). The sequence $W_{n}^{\prime R}$ has the advantage of eliminating phase shifters (since all the weighting coefficients are real) at the cost of increasing the number of elements in the array from $M$ to $2 M-1$.

\section{MODE FILTERING WITH A NON-HORIZONTAL ARRAY}

In order to determine the response of a nonhorizontal array with equispaced elements, we have to substitute Eq. (10) into Eq. (16). The array gain factor for mode $m$ is given by

$$
\begin{aligned}
g_{m}= & \sum_{q} a_{m} w_{q} \sin \left[\gamma_{m}\left(z_{0}+q d \cos \alpha\right)\right] \\
& \times \exp \left(-j k_{m} q d \sin \alpha \cos \beta\right) .
\end{aligned}
$$

In order to render $g_{n}=0$ for all $z_{0}$, we need at least three weighting coefficients to be nonzero. It can be readily verified that $g_{m}=0$ if we assign nonzero weights to three consecutive elements $p-1, p, p+1$ so that

$w_{p}=1$,

$$
\begin{aligned}
w_{p+1} & =w_{p}^{*}-1 \\
& =-\frac{1}{2} \sec \left(\gamma_{m} d \cos \alpha\right) \exp \left(j k_{m} d \sin \alpha \cos \beta\right),
\end{aligned}
$$

and assign zero weights to all the other elements. Hence, for a slanted array, the weight sequence required to reject mode $m$ can be written as

$$
\begin{aligned}
U_{m}^{S}= & \left\{-\frac{1}{2} \sec \left(\gamma_{m} d \cos \alpha\right) \exp \left(-j k_{m} d \sin \alpha \cos \beta\right),\right. \\
& \left.1,-\frac{1}{2} \sec \left(\gamma_{m} d \cos \alpha\right) \exp \left(j k_{m} d \sin \alpha \cos \beta\right)\right\} .
\end{aligned}
$$

The response of the array defined by Eq. (31) to a plane wave incident at an angle $\theta$ can be oblained using Eq. (24). The response is given by

$$
\begin{aligned}
B_{m}(\theta)= & 1-\sec \left(\gamma_{m} d \cos \alpha\right) \cos \left(k_{m} d \sin \alpha \cos \beta\right. \\
& -k d \cos \theta) .
\end{aligned}
$$

The zeros of $B_{a n}$ are given by

$$
\theta_{m}=\cos { }^{1}\left[(1 / k)\left(k_{m} \sin \alpha \cos \beta \pm \gamma_{m} \cos \alpha\right)\right],
$$

which are precisely the directions of arrival (with respect to the array axis) of the two modal waves associated with mode $m$.

Filtering of mode $n$ can be achieved by using a ( $2 M$ -1 ) element array whose weight sequence $W_{n}^{S}$ is obtained by substituting $U_{m}=U_{i n}^{S}(m=1, \ldots, n-1, n+1, \ldots M)$ in Eq. (18).

The elementary weight sequence $U_{m}^{s}$ defined by $\mathrm{Eq}$. (31) and the corresponding sequence $W_{n}^{W}$ obtained from Eq. (18) are Hermitian, i.e., weights of the elements equidistant from the central element on either side are complex conjugates of one another. However, for a vertical array, the angle $\alpha$ is zero and the weights in the sequence $U_{m}^{s}$ become real. Hence, for a vertical array, the sequences $U_{m}^{S}$ and $W_{n}^{S}$ are real and symmetric. Putting $\alpha=0$ in Eq. (31) and res- 
caling, we can write the weight sequence of the elementary vertical array for rejecting the $m$ th mode as

$$
U_{m}^{v}=\left\{1,-2 \cos \left(\gamma_{m} d\right), 1\right\} .
$$

Convolution of $M-1$ such sequences gives the weight sequence $W_{n}^{V}$ of the $n$th mode filter. Comparison of Eq. (34) ( with Eq. (28) indicates that the weight sequence of the vertical array mode filter is similar to that of the horizontal end fire array mode filter $(\beta=0)$, with the horizontal wave number $k_{m}$ replaced by the vertical wave number $\gamma_{m}$.

The sequence $W_{n}^{S}$ defined by Eqs. (31) and (18) can be used for mode filtering with a horizontal array $(\alpha=\pi / 2)$ also. However, for a horizontal array, it would be better to use either the weight sequence $W_{n}^{H}$ obtained by convolving the elementary sequences $U_{m}^{H}$ [Eq. (23)] or the weight sequence $W_{n}^{H R}$ obtained by convolving the elementary sequences $U_{m}^{H R}$ [Eq. (28)]. The sequence $W_{n}^{H}$ has the advantage of smaller array length ( $M$ elements instead of $2 M-1$ ), while the sequence $W_{n}^{H R}$ has the advantage of real and symmetric weighting coefficients.

\section{ADVANTAGES OF THE NULL-STEERING TECHNIQUE}

Let $w_{n q},(n=1, \ldots M$ and $q=1, \ldots, Q)$ denote the $q$ th weighting coefficient of the $n$th mode filter, and let $g_{n m}$ denote the gain factor of the $n$th mode filter for the $m$ th mode input. Following Eq. (16), we can write

$$
\begin{aligned}
g_{n m}= & \sum_{q}^{Q} a_{m} w_{n q} \sin \left[\gamma_{m}\left(q d \cos \alpha+z_{0}\right)\right] \\
& \times \exp \left(-j k_{m} q d \sin \alpha \cos \beta\right) .
\end{aligned}
$$

The output of the $n$th mode filter is given by

$$
R_{n}=\sum_{m=1}^{M} g_{n i m} A_{m} \text {. }
$$

Using the null-steering technique, $w_{n q}$ can be chosen in such a way that $g_{n n}=0$ for $m \neq n$. The output of the null-steering mode filter is therefore given by

$$
R_{n}=g_{n n} A_{n} \text {. }
$$

The output can be made equal to the mode amplitude $A_{n}$ if the weighting coefficients are normalized so as to render $g_{n n}=1$.

The foregoing discussion highlights the fact that the null-steering technique provides, for the first time, a method of achieving perfect mode filtering (zero leakage from the unwanted modes) by employing either a vertical or a horizontal $(2 M-1)$-element equispaced array with real weighting coefficients. Furthermore, this method does not impose any constraint either on the mean array depth $z_{0}$ or the interelement spacing $d$, except that a horizontal array filter for the $n$th mode should not be located at a depth close to a node of the $n$th mode. On the other hand, some leakage is invariably present when the mode-matching method using a vertical array or the least-squares method using either a vertical or a horizontal array are employed. To minimize the leakage, the mode matching and least squares techniques require the deployment of a vertical array that spans the entire depth of the channel, whereas good filtering can be achieved with short vertical arrays placed anywhere in the channel if the null-steering technique is used.

Another major advantage of the null-steering technique stems form the fact that the weighting coefficients are independent of the mean array depth. Hence, the performance of the null-steering mode filter is not affected by variations in the array depth. It is pertinent to note that it is very difficult to avoid variations in the array depth at sea and these variations adversely affect the performance of other types of mode filters.

The null-steering mode filter performs equally well under all bottom conditions, whereas the mode-matching filter performs poorly in a Perkeris channel where the lower-order mode functions have significant penetration into the sediment.

Even though the analysis in Secs. II and III is based on the assumption of isovelocity water channel, the isovelocity constraint is required only for a nonhorizontal array. For a horizontal array, the elementary weighting sequence $U_{m}^{H}$ given by Eq. (23) can be used to reject the $m$ th mode even when the channel is horizontally stratified. The gain factor $g_{n n}$ of the mode filter in a horizontally stratified channel is obtained by replacing the factor $a_{m} \sin \left(\gamma_{m} z_{0}\right)$ in Eq. (35) by the appropriate mode function $\psi_{m}\left(z_{0}\right)$.

The weighting coefficients of a slanted array mode filter depend on the angles $\alpha$ and $\beta$, the wave numbers $\gamma_{m}$ and $k_{m}$, and the interelement spacing $d$ [see Eq. (31) ]. However, it is sufficient to know the values of the parameters $\gamma_{m}$ and $d$ for a vertical array [Eq. (34)], and the parameters $k_{m} \cos \beta$ and $d$ for a horizontal array [Eq. (23)]. The issue of sensitivity of the null-steering mode filter to errors in the assumed values of these parameters is discussed in Sec. VI.

\section{NUMERICAL SIMULATION}

The performance of the null-steering mode filter has been tested by computer simulation for isovelocity channels with three types of bottom conditions, viz., a rigid bottom, a pressure-release bottom, and a fluid bottom (Pekeris channel), and it has been verified that perfect filtering can be achieved in each case. Details of the simulation results for a Pekeris channel are presented below.

Numerical values assigned to the parameters in the computation are as follows: water depth $h=50 \mathrm{~m}$, source depth $z_{\mathrm{s}}=25 \mathrm{~m}$, source frequency $f=60 \mathrm{~Hz}$, sound speed in water $c=1500 \mathrm{~m} / \mathrm{s}$, sound speed in sediment $c_{1}=2000$ $\mathrm{m} / \mathrm{s}$, density of water $\rho=1000 \mathrm{~kg} / \mathrm{m}^{3}$, density of sediment $\rho_{1}=1100 \mathrm{~kg} / \mathrm{m}^{3}$. The modal eigenvalues $\gamma_{m}$ are the roots of the equation ${ }^{16}$

$\rho_{1} \gamma_{m} \cot \left(\gamma_{m} h\right)+\rho\left\{k^{2}\left[1-\left(c / c_{1}\right)^{2}\right]-\gamma_{m}^{2}\right\}^{1 / 2}=0$

and the normalization constants $a_{t m}$ are given by

$$
\begin{aligned}
a_{m}^{2}= & \frac{2}{h}\left\{1-\left(\frac{\rho}{\rho_{1}}\right)^{2}\left(\frac{k^{2}}{\gamma_{m}^{3} h}\right)\left[1-\left(\frac{c}{c_{1}}\right)^{2}\right]\right. \\
& \left.\times \sin ^{2}\left(\gamma_{m} h\right) \tan \left(\gamma_{m} h\right)\right\}^{-1} .
\end{aligned}
$$

For the assigned parameter values there are three propagating modes in the channel, and the angles between the directions of propagation of the modal plane waves and the horizontal plane are given by $\pm 12.7375^{\circ}, \pm 26.0107^{\circ}$, and 
TABLE I. Vertical array weighting coefficients.

\begin{tabular}{cccc}
\hline \hline $\begin{array}{c}\text { Mode No. } \\
n\end{array}$ & $w_{n 1}=w_{n 5}$ & $w_{n 2}=w_{n 4}$ & $w_{n 1}$ \\
\hline 1 & 1.0000 & -0.8157 & 1.9209 \\
2 & 1.0000 & -1.6131 & 1.8510 \\
3 & 1.0000 & -2.6040 & 3.5363 \\
\hline \hline
\end{tabular}

$\pm 39.9738^{\circ}$. The field at the individual array elements is computed using Eq. (11).

\section{A. Vertical array}

A five-element equispaced array with interelement spacing $d=10 \mathrm{~m}$ is set at a range of $1 \mathrm{~km}$ from the source. The array center is located at a depth $z_{0}=25 \mathrm{~m}$. The modal wave arrival directions with respect to the array axis are given by $\theta_{1}=90^{\circ} \pm 12.7375^{\circ}, \quad \theta_{2}=90^{\circ} \pm 26.0107^{\circ}$, $\theta_{3}=90^{\circ} \pm 39.9738^{\circ}$. The corresponding mode filter weighting coefficients computed using Eqs. (34) and (18) are given in Table I. In Table I, $w_{n q}$ denotes the $q$ th weighting coefficient of the $n$th filter. The free-field directivity functions of the mode filters are shown in Fig. 2. If the filtering is perfect, one expects the mode filter output to be inversely proportional to the square root of range $r$. Filter outputs for each mode indeed follow the expected variation as shown by the thick lines in Fig. 3. Also shown in Fig. 3 are the results of filtering when the mode-matching technique is used with the same number of elements. In the latter results, one can see oscillations about the ideal $1 / \sqrt{r}$ variation due to leakage from the unwanted modes. Also perfect filtering can be achieved using the null-steering technique for any choice of values of $z_{0}$ and $d$. On the other hand, the performance of the mode-matching technique, for a given number of elements in the array, is dependent on the depths at which the elements are located. A proper choice of the element depths is necessary to minimize leakage with the mode-matching technique.

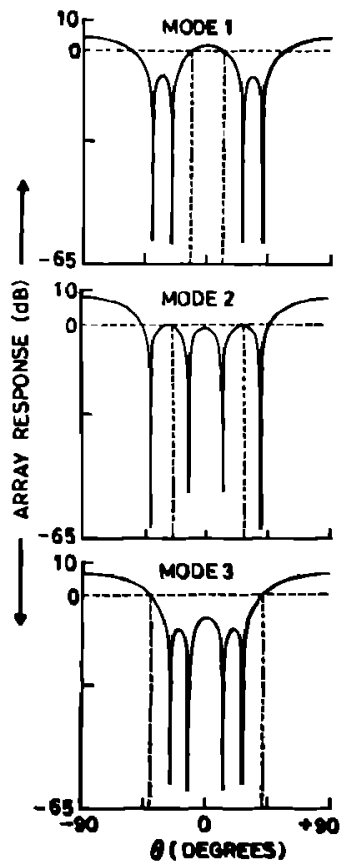

FIG. 2. Free-field directivity functions of the vertical array mode filters. The response is normalized with respect to the amplitude in the directions of arrival of the filtered (selected) modal waves.

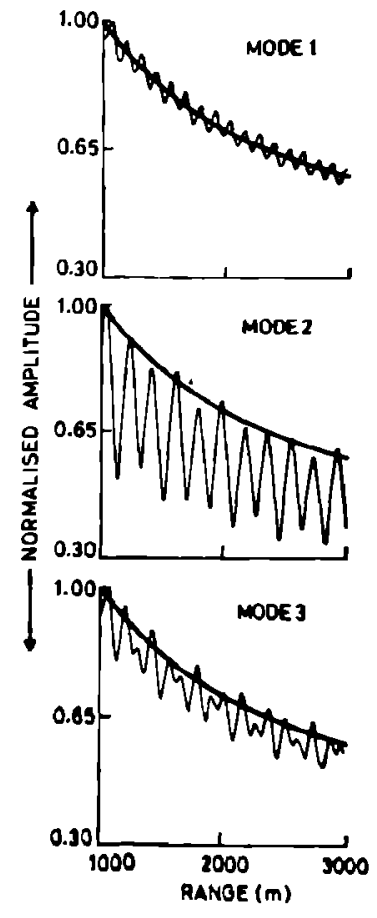

FIG. 3. Comparison of mode filter output as a function of range obtained using the null-steering technique (thick line) and the mode-matching technique (thin line).

\section{B. Horizontal array}

We have also considered a horizontal end-fire array, with an interelement spacing $d=10 \mathrm{~m}$, located at a depth $z_{0}=25 \mathrm{~m}$. A three-element horizontal array is adequate to filter one of the three modes. In this case, the mode filter weighting coefficients (Table II) are computed from Eqs. (23) and (18) with $\beta=0$, and the corresponding free-field directivity functions are shown in Fig. 4. Ideal filter performance is obtained in this case also. Note that the weighting coefficients are complex in this case. As stated in Sec. III, an array with real weighting coefficients can be designed by adding two elements to the array and steering two additional nulls. The real sequence $W_{n}{ }^{I R}$ can be obtained by convolving the complex sequence $W^{\prime \prime}$ "with its complex conjugate. Values of these real weighting coefficients are given in Table III.

\section{SENSITIVITY ANALYSIS}

The weighting coefficients of the null-steering mode filter depend upon the interelement spacing $d$, the wave numbers $\gamma_{m}$ and $k_{m}$, and the angles $\theta_{m}$ between the array axis and the modal plane wave arrival directions. The angles $\theta_{m}$, in turn, depend on the angles $\alpha$ and $\beta$. Hence, any discrepancy between the actual and the assumed values of the parameters listed above can degrade the performance of the mode filter due to the leakage of unwanted modes into the

TABLE II. Horizontal end-fire array weighting coefficients. Three-element array with complex weighting coefficients.

\begin{tabular}{cccc}
\hline \hline $\begin{array}{c}\text { Mode No. } \\
n\end{array}$ & $w_{n 1}$ & $w_{u}$ & $\omega_{n+}$ \\
\hline 1 & 1.0000 & $0.9827+j 1.7101$ & $-0.5035+j .8640$ \\
2 & 1.0000 & $1.1189+j 1.5742$ & $-0.3287+j .9444$ \\
3 & 1.0000 & $1.4061+j 1.4092$ & $-0.0023+j 1.0000$ \\
\hline
\end{tabular}




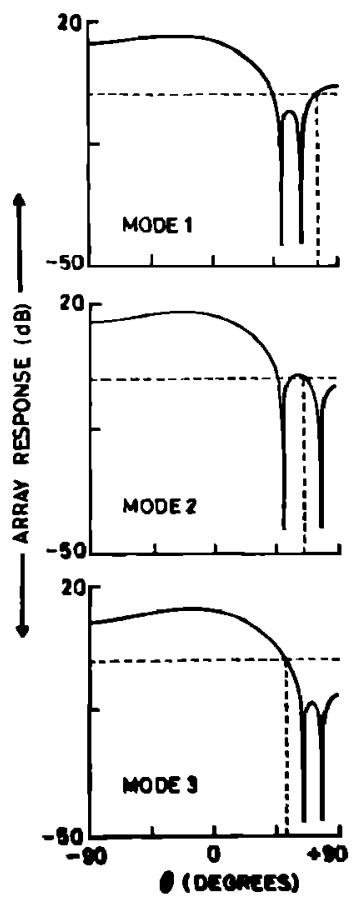

FIG. 4. Free-field directivity functions of the horizontal array mode filters. The response is normalized with respect to the amplitude in the direction of arrival of the filtered (selected) modal wave.

mode filter output. The value of the parameter $d$ may be known with sufficient accuracy, but errors in the assumed values of $\alpha$ and $\beta$ may occur due to practical difficulties in maintaining a steady orientation of the array. Errors may also arise in the assumed or estimated values of the environmental parameters $\gamma_{m}$ and $k_{m}$. Sensitivity of the null-steering mode filter to these errors is discussed below.

We define the mode leakage factor $\lambda_{n m}$ as

$$
\lambda_{n m}=10 \log \left(\left|g_{n m}\right| /\left|g_{n n}\right|\right) \text {, }
$$

where $g_{n m}$ is the gain factor defined in Eq. (35). The quantity $\lambda_{n m}$ denotes the leakage of mode $m$ into the output of the mode- $n$ filter. For null-steering mode filter, $\lambda_{n m}=0$ for $m \neq n$, if the parameters $d, \alpha, \beta, \gamma_{m}$, and $k_{m}$ are known accurately.

The effect of tilt of a vertical array from its assumed vertical position is shown in Figs. 5 and 6 . In these figures, the leakage factors $\lambda_{n m}$ of the vertical array considered in Sec. $V$ are plotted as functions of the angle of tilt $\delta$. In Fig. 5, the plane of tilt coincides with the vertical plane containing the source and the array center, i.e., the plane of tilt is the $x z$ plane with $\alpha=\delta, \beta=0$. In Fig. 6, the plane of tilt is perpendicular to the vertical plane containing the source and the array center, i.e., the plane of tilt is the $y z$ plane with $\alpha=\delta$, $\beta=\pi / 2$. Similar plots for the horizontal end-fire array considered in Sec. $V$ are shown in Figs. 7 and 8. In Fig. 7, the

TABLE III. Horizontal end-fire array weighting coefficients. Five-element array with real weighting coefficients.

\begin{tabular}{cccc}
\hline \hline Mode No. & \multicolumn{3}{c}{ Weighting coefficients } \\
$n$ & $w_{n 1}=w_{n 5}$ & $w_{n 2}=w_{n 4}$ & $w_{n 3}$ \\
\hline 1 & 1.0000 & 1.9655 & 2.8833 \\
2 & 1.0000 & 2.2379 & 3.0728 \\
3 & 1.0000 & 2.8121 & 3.9585 \\
\hline
\end{tabular}

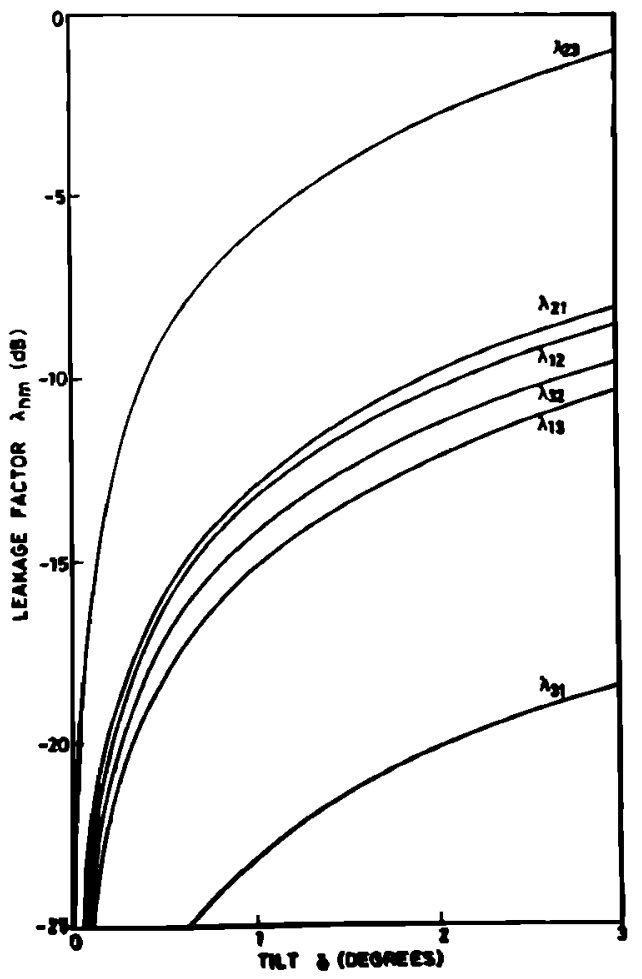

FIG. 5. Variation of mode leakage factors $\lambda_{u m}$ of the vertical array as a function of tilt $\delta$ in the $x z$ plane: $d=10 \mathrm{~m}, z_{0}=25 \mathrm{~m}, f=60 \mathrm{~Hz}$.

plane of tilt is vertical, i.e., the plane of tilt is the $x z$ plane with $\alpha=\pi / 2 \pm \delta, \beta=0$. In Fig. 8, the plane of tilt is horizontal, i.e., the plane of tilt is the $x y$ plane with $\alpha=\pi / 2$, $\beta=\delta$. Figures $5-8$ indicate that the mode filter is considerably more sensitive to tilts in the $x z$ plane as compared to tilts

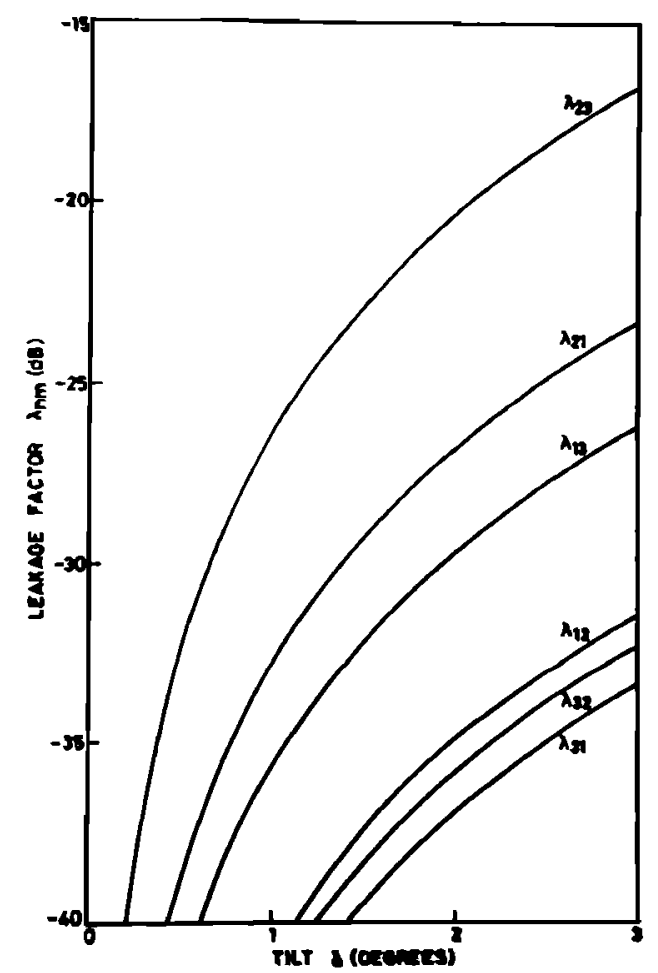

FIG. 6. Variation of mode leakage factors $\lambda_{n m}$ of the vertical array as a function of tilt $\delta$ in the $y z$ plane: $d=10 \mathrm{~m}, z_{0}=25 \mathrm{~m}, f=60 \mathrm{~Hz}$. 


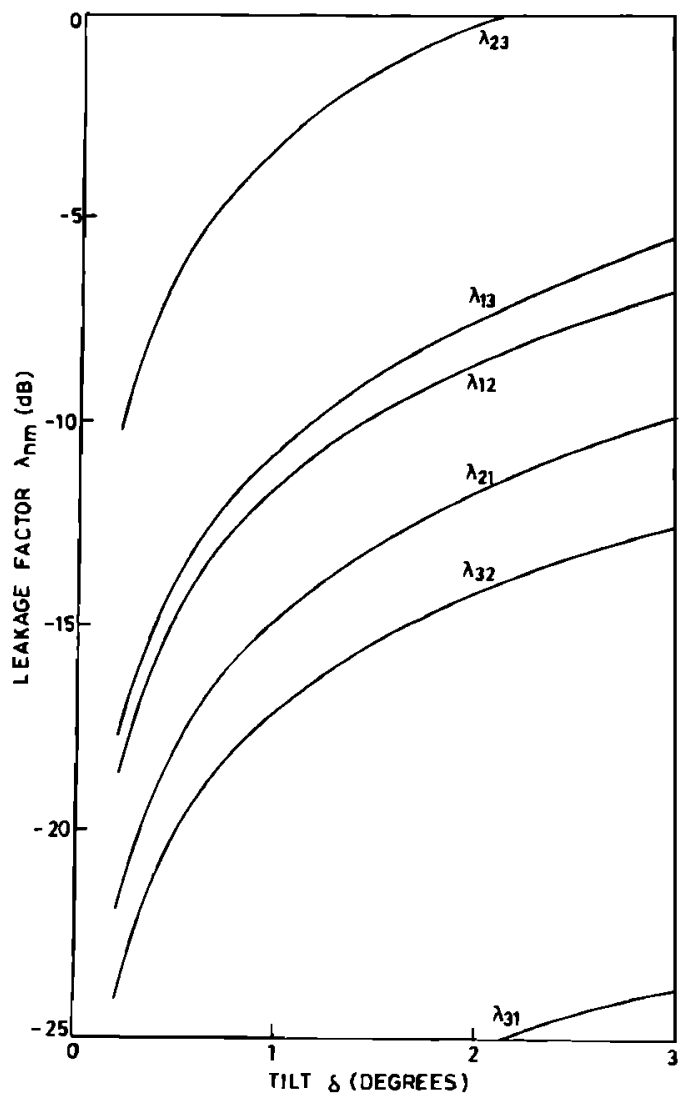

FIG. 7. Variation of mode leakage factors $\lambda_{m m}$ of the horizontal end-fire array as a function of tilt $\delta$ in the $x z$ plane: $d=10 \mathrm{~m}, z_{0}=25 \mathrm{~m}, f=60 \mathrm{~Hz}$.

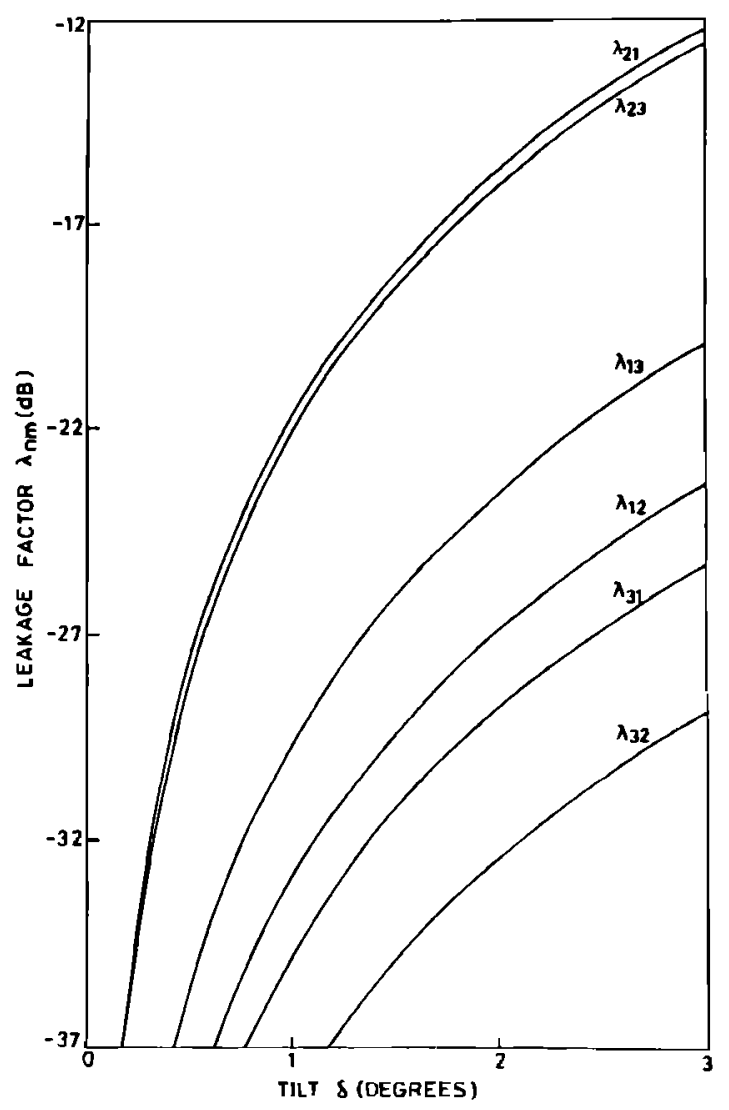

FIG. 8. Variation of mode leakage factors $\lambda_{n m}$ of the horizontal end-fire array as a function of tilt $\delta$ in the $x y$ plane: $d=10 \mathrm{~m}, z_{0}-25 \mathrm{~m}, f=60 \mathrm{~Hz}$. either in the $y z$ plane (in the case of a vertical array) or in the $x y$ plane (in the case of a horizontal array). This difference in sensitivity is due to the fact that a tilt of the array through an angle $\delta$ in the $x z$ plane causes the angles $\theta_{m}$ between the array axis and the direction of travel of the modal plane waves also to change by the same amount $\delta$. On the other hand, the same degree of tilt either in the $y z$ plane or in the $x y$ plane causes the angles $\theta_{m}$ to change by much smaller amounts.

As already stated, the weighting coefficients of the vertical array mode filter depend on the environmental parameters $\gamma_{m}$ only, while those of the horizontal array mode filter depend on the environmental parameters $k_{m}$ only. Leakage factors of the vertical and horizontal endfire array considered above are plotted as functions of the percentage error in $\gamma_{m}$ and $k_{m}$, respectively, in Figs. 9 and 10. It appears that the vertical array is less sensitive to error in the environmental parameters than the horizontal array. But this conclusion cannot be generalized since the sensitivity also depends on the choice of the other parameters such as $d$ and $z_{0}$.

The sensitivity of the mode filter to errors in orientation or environmental parameters can be minimized by an optimal choice of $d$ and $z_{0}$. The variation of sensitivity with $d$ and $z_{0}$ is illustrated in Figs. 11-16. In these figures, $\lambda_{n}$ is a measure of total leakage of all unwanted modes into the output of mode- $n$ filter, defined as

$$
\lambda_{n}=10 \log \left\{\left.\sum_{m \neq n} g_{n m}\right|^{2}\right\}^{1 / 2}\left(\left|g_{n n}\right|\right) \quad \text { '. }
$$

In Eq. (41) an incoherent summation is preferred to a coherent summation since the phase of $g_{n m}$ would be randomly distributed. For a vertical array, the variation of $\lambda_{n}$ with $d$

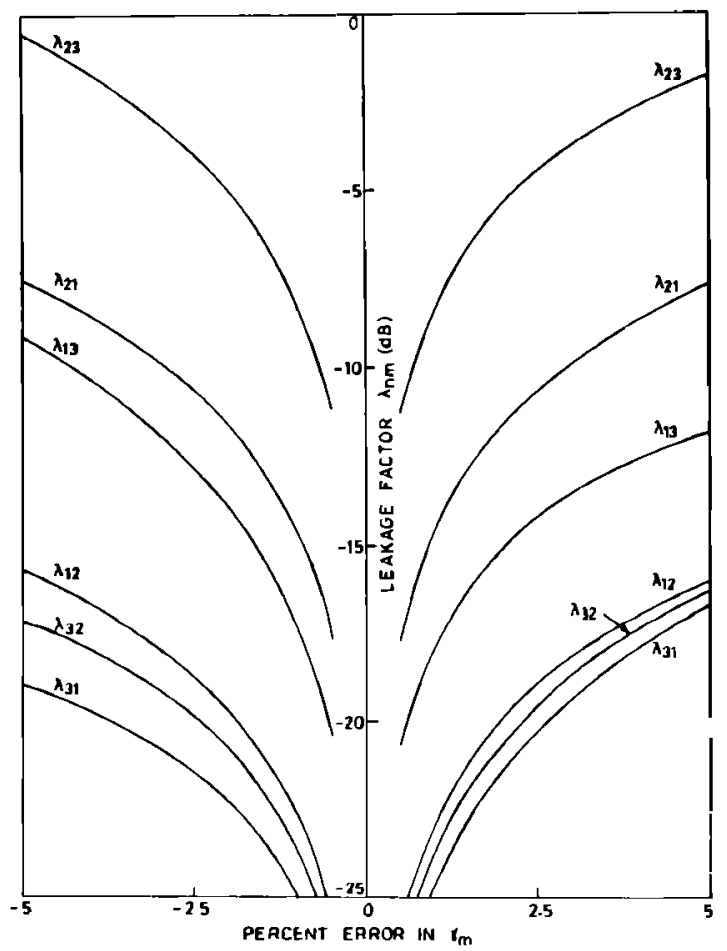

FIG. 9. Variation of mode leakage factors $\lambda_{\text {nem }}$ of the vertical array as a function of percentage error in $\gamma_{m}: d=10 \mathrm{~m}, z_{11}-25 \mathrm{~m}, f-60 \mathrm{~Hz}$. 


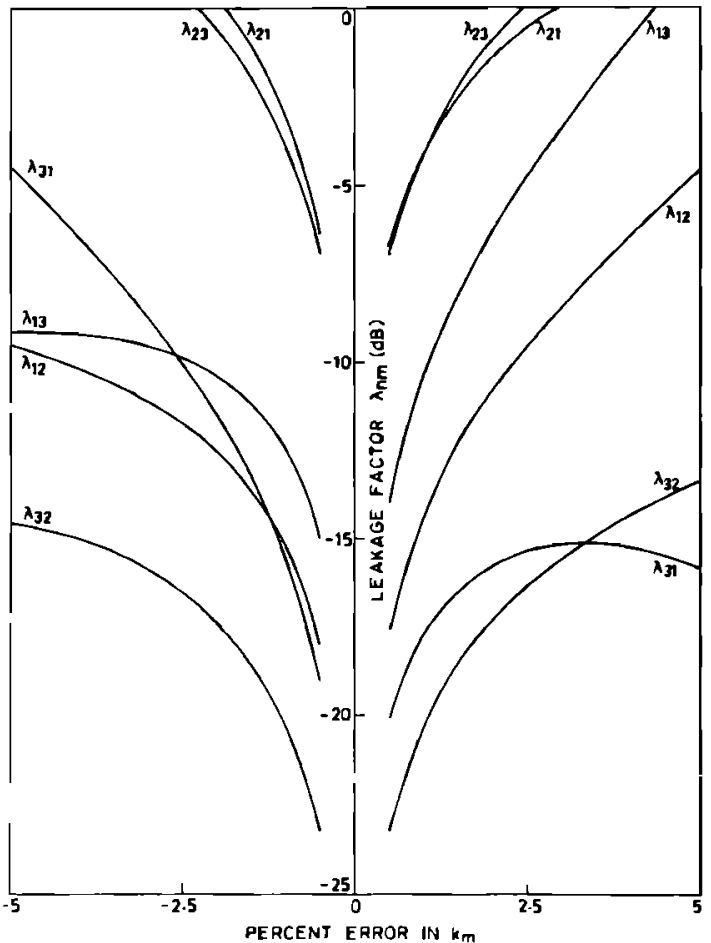

FIG. 10. Variation of mode leakage factors $\lambda_{\text {uाn }}$ of the horizontal end-fire array as a function of percentage error in $k_{\mathrm{u},}: d=10 \mathrm{~m}, z_{0}=25 \mathrm{~m}, f=60$ Hz.

for a $1^{\circ}$ tilt in the $x z$ plane and for $1 \%$ error in all the $\gamma_{m}$ 's is shown in Figs. 11 and 12, respectively. In both cases, the largest of the $\lambda_{n}$ 's decreases monotonically as $d$ is increased. Numerical computations indicate that this result is true in general for all vertical arrays. Hence, the interelement spacing $d$ of a vertical array should be made as large as possible to minimize leakage. Since the number of elements in the array is $2 M-1$, the maximum possible value of $d$ is equal to $h / 2(M-1)$, where $h$ is the depth of the channel.

The dependence of $\lambda_{n}$ on $d$ and $z_{0}$ is considerably more complex in the case of a horizontal array. Typical plots of $\lambda_{n}$ vs $d$ and $\lambda_{n}$ vs $z_{0}$ for a horizontal end-fine array with a $1^{\circ}$ tilt

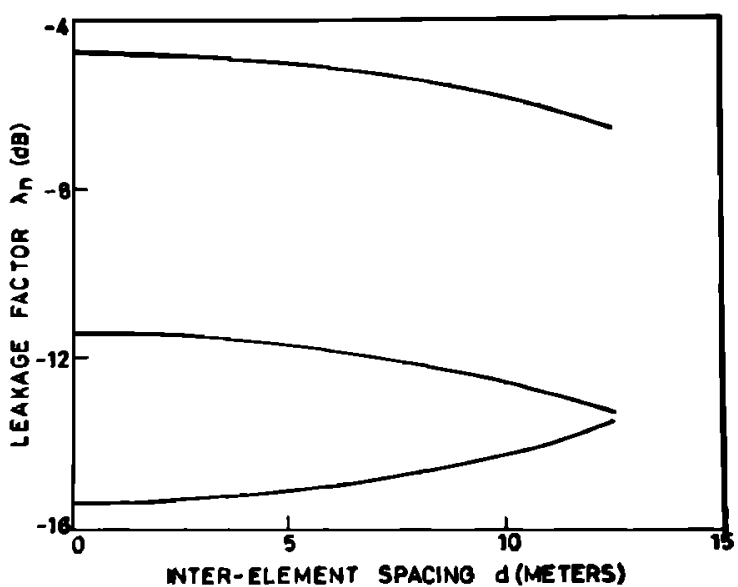

FIG. 11. Variation of mode leakage factors $\lambda_{n}$ of the vertical array as a function of interelement spacing $d$ for $1^{\circ}$ tilt in the $x z$ plane: $z_{0}=25 \mathrm{~m}$, $f=60 \mathrm{~Hz}$.

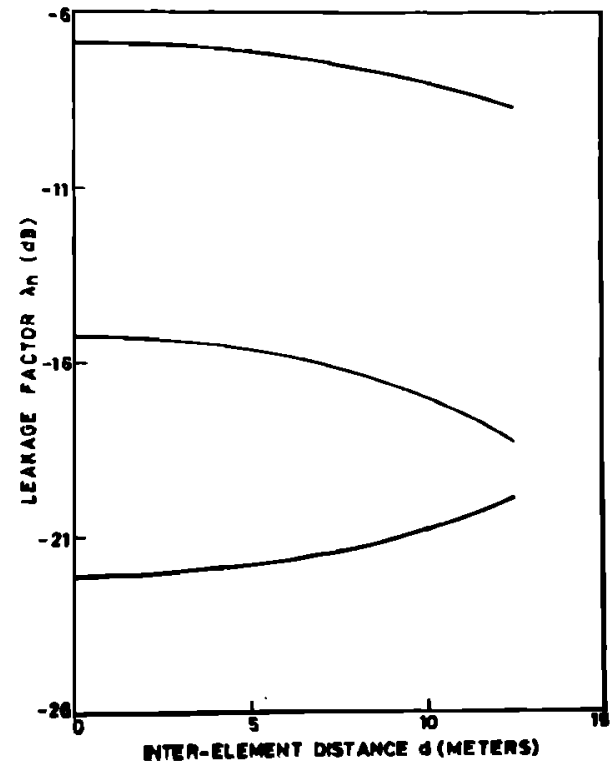

FIG. 12. Variation of mode leakage factors $\lambda_{n}$ of the vertical array as a function of interelement spacing $d$ for $1 \%$ error in all $\gamma_{m}{ }^{\prime} \mathrm{s}: z_{0}=25 \mathrm{~m}$, $f=60 \mathrm{~Hz}$.

in the $x z$ plane are shown in Figs. 13 and 14. These figures indicate low sensitivity to tilt for $d \simeq 10$ or $22 \mathrm{~m}$ and $z_{0} \simeq 10$ $\mathrm{m}$. Extensive numerical computations indicate that this choice of $d$ and $z_{0}$ is close to optimum. Optimum values are those values of $d$ and $z_{0}$ for which the largest of the $\lambda_{n}$ 's is minimum. Unlike the case of a vertical array, there is no upper limit on the value of $d$ due to physical constraints in the case of a horizontal array. But practical considerations rule out the use of very large values of $d$.

Typical plots of $\lambda_{n}$ vs $d$ and $\lambda_{n}$ vs $z_{0}$ for a horizontal endfire array with $1 \%$ error in all the $k_{m}$ 's are shown in Figs. 15 and 16. It is seen that the choice of $d \simeq 10$ or $22 \mathrm{~m}$ and $z_{0} \simeq 41$ m offers low sensitivity to errors in $k_{m}$.

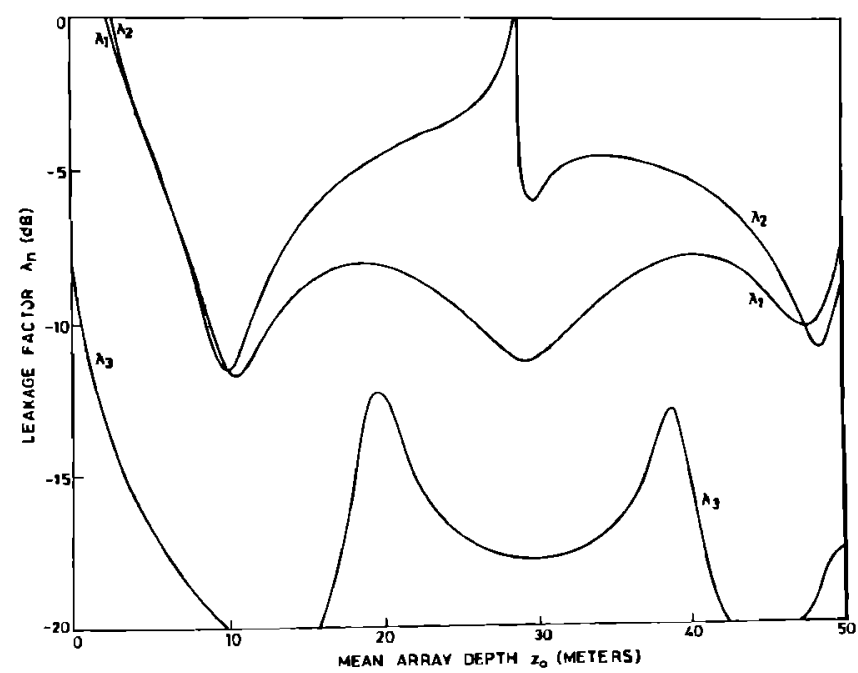

FIG. 13. Variation of mode leakage factors $\lambda_{n}$ of the end-fire horizontal array as a function of array depth $z_{0}$ for $1^{\circ}$ tilt in the $x z$ plane. $d=10 \mathrm{~m}$, $f=60 \mathrm{~Hz}$. 


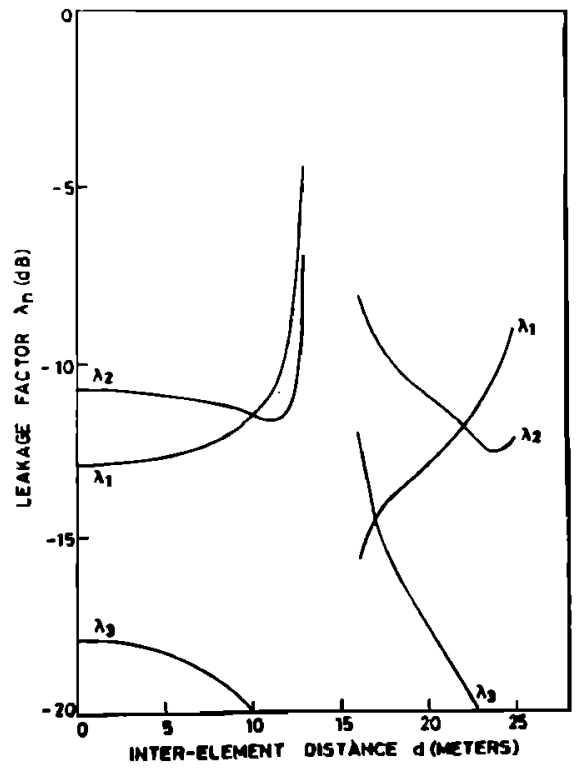

FIG. 14. Variation of mode leakage factors $\lambda_{n}$ of the end-fire horizontal array as a function of interelement spacing $d$ for $1^{\circ}$ tilt in the $x z$ plane: $z_{0}=10 \mathrm{~m}, f=60 \mathrm{~Hz}$.

\section{CONCLUSIONS}

The null-steering technique of mode filtering presented in this paper offers several advantages. The foremost is that the array weighting coefficients do not depend on the depth at which the array is deployed. Hence the performance of the filter is not affected by changes in the position of the array, as long as its orientation is maintained. Secondly, there is no constraint on the interelement spacing and it is not necessary to use an array spanning the entire depth of the channel. Equally good performance can be obtained with all types of bottom. For example, unlike the mode-matching technique, perfect filtering is achieved even when the mode functions have a significant penetration into the bottom.

A null-steering mode filter can use either a vertical or a horizontal array of $2 M-1$ elements with real weighting coefficients. For a horizontal array, the number of elements

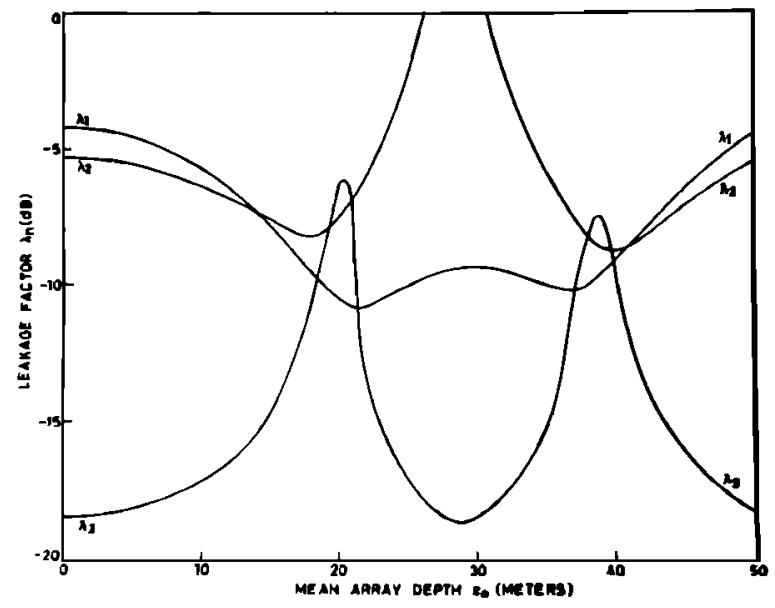

FIG. 15. Variation of mode leakage factors $\lambda_{n}$ of the end-fire horizontal array as a function of array depth $z_{11}$ for $1 \%$ error in all $k_{m}$ 's: $d=10 \mathrm{~m}$, $f=60 \mathrm{~Hz}$.

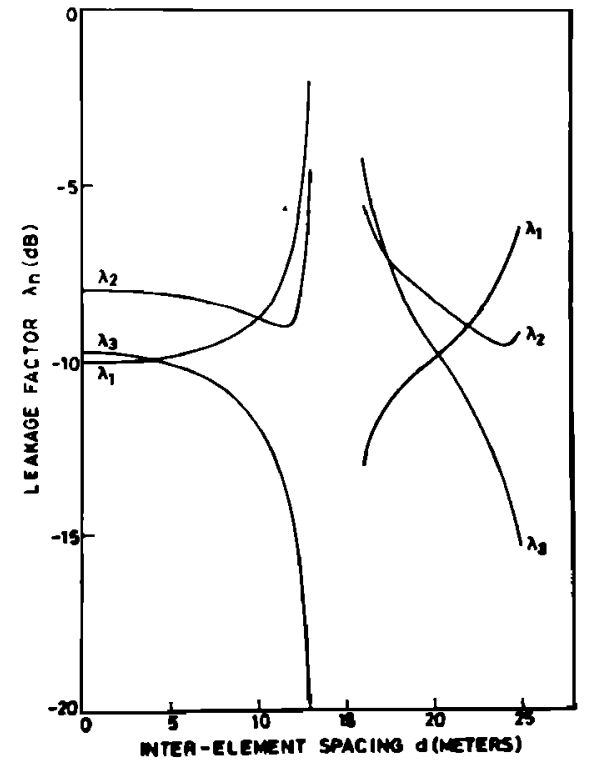

FIG. 16. Variation of mode leakage factors $\lambda_{\text {n }}$ of the end-fire horizontal array as a function of interelement spacing $d$ for $1 \%$ error in all $k_{m}$ 's: $z_{\mathrm{i}}-41 \mathrm{~m}, f-60 \mathrm{~Hz}$.

can be reduced to $M$ if complex weighting coefficients are used. The horizontal array can be used in a horizontally stratified channel also, whereas the use of a null-steering vertical array mode filter is restricted to isovelocity channels. When a horizontal array is used, care should be taken to ensure that (1) the source is in or near the end-fire direction, and (2) the array is not deployed at a depth close to the node of the mode required to be filtered.

A null-steering vertical array requires the knowledge of the vertical wave numbers $\gamma_{m}$, while a null-steering horizontal array requires the knowledge of the horizontal wave numbers $k_{m}$. In an unknown channel, the horizontal wave numbers $k_{m}$ may be estimated using Prony's method as shown by Shang et al. ${ }^{4}$ Two other methods of estimating $k_{m}$, using short arrays, are described in the Ph.D. thesis of the first author. ${ }^{17}$

Errors in the assumed array orientation or the assumed modal wave numbers can lead to degradation of the filter performance. The filter is considerably more sensitive to tilts in the $x z$ plane (vertical plane containing the source and the array center) than to tilts in the $y z$ or $x y$ planes. The sensitivity to errors in the assumed values of $\alpha, \beta, \gamma_{m}$, and $k_{m}$ can be minimized by an optimum choice of the interelement spacing $d$ and array depth $z_{0}$.

Details of the hardware implementation of the mode filtering processor based on the null-steering technique and its use in model studies in a Laboratory tank can be found in Ref.17.

\section{ACKNOWLEDGMENT}

This work was supported by the Department of Electronics, Government of India.

'F. Ingenito, “Measurement of mode attenuation coeficients in shallow
water," J. Acoust. Soc. Am. 53. 858863 (1973)
${ }^{2}$ C. T. Tindle, “Measurement of the frequency dependence of the normal
mode," J. Acoust. Soc. Am. 64. 1178-1185 (1978).
${ }^{1}$ E. C. Lo, J. X. Zhou, and E. C. Shang, "“Normal mode filtering in shallow 
water," J. Acoust. Soc. Am. 74, 1833-1836 (1983).

${ }^{4}$ E. C. Shang, H. P. Wang, and Z. Y. Huang, "Waveguide characterization and source localization in shallow water waveguides using the Prony method," J. Acoust. Soc. Am. 83, 103-108 (1988).

${ }^{5}$ E. C. Shang, "Source depth estimation in waveguides," J. Acoust. Soc. Am. 77, 1413-1418 (1985).

${ }^{6}$ E. C. Shang, C. S. Clay, and Y. Y. Wang, "Passive harmonic source ranging in waveguide by using mode filter," J. Acoust. Soc. Am. 78, 173-176 (1985).

${ }^{7}$ T. C. Yang, "A method of range and depth estimation by modal decomposition," J. Acoust. Soc. Am. 82, 1736-1745 (1987).

${ }^{8}$ G. R. Wilson, R. A. Koch, and P. J. Vidmar, "Matched mode localization," J. Acoust. Soc. Am. 84, 310-320 (1988).

${ }^{9}$ E. C. Shang, "An efficient high-resolution method of source localization processing in mode space," J. Acoust. Soc. Am. 86, 1960-1964 (1989).

${ }^{10} \mathrm{E}$. C. Shang, "Ocean acoustic tomography based on adiabatic mode theory," J. Acoust. Soc. Am. 85, 1531-1537 (1989).
${ }^{1}$ R. H. Ferris, "Comparison of measured and calculated normal mode amplitude functions for acoustic waves in shallow water," J. Acoust. Soc. Am. 52, 981-988 (1972).

${ }^{12}$ C. S. Clay, "Array steering in a layered waveguide," J. Acoust. Soc. Am. 33, 865-870 (1961).

${ }^{13}$ C. S. Clay, "Waveguide, arrays, filters," Geophysics 31, 501-505 (1966).

${ }^{14} \mathrm{C}$. S. Clay and H. Medwin, Acoustical Oceanography: Principles and Applications (Wiley-Interscience, New York, 1977), pp. 494-497.

${ }^{15} \mathrm{C}$. Gazanhes, J. P. Sessarego, and J. L. Garnier, "Identification of modes in some conditions of sound propagation in shallow water," J. Sound. Vib. 56, 251-259 (1978).

${ }^{16} \mathrm{~L}$. Brekhovskikh and Y. Lysanov, Fundamentals of Ocean Acoustics (Springer-Verlag, New York, 1982), pp. 101-102.

${ }^{17}$ H. M. Chouhan, "Normal Mode Decomposition and Its Applications in Ocean Acoustics," Ph.D. thesis, Department of Electrical Communication Engineering, Indian Institute of Science, Bangalore, India (1989), Chap. 5. 